Internet Engineering Task Force (IETF)

Request for Comments: 6169

Category: Informational

ISSN : 2070-1721
S. Krishnan

Ericsson

D. Thaler

Microsoft

J. Hoagland

Symantec

April 2011

\title{
Security Concerns with IP Tunneling
}

\section{Abstract}

A number of security concerns with IP tunnels are documented in this memo. The intended audience of this document includes network administrators and future protocol developers. The primary intent of this document is to raise the awareness level regarding the security issues with IP tunnels as deployed and propose strategies for the mitigation of those issues.

Status of This Memo

This document is not an Internet Standards Track specification; it is published for informational purposes.

This document is a product of the Internet Engineering Task Force (IETF). It represents the consensus of the IETF community. It has received public review and has been approved for publication by the Internet Engineering Steering Group (IESG). Not all documents approved by the IESG are a candidate for any level of Internet Standard; see section 2 of RFC 5741.

Information about the current status of this document, any errata, and how to provide feedback on it may be obtained at http://www.rfc-editor.org/info/rfc6169.

Copyright Notice

Copyright (c) 2011 IETF Trust and the persons identified as the document authors. All rights reserved.

This document is subject to BCP 78 and the IETF Trust's Legal Provisions Relating to IETF Documents (http://trustee.ietf.org/license-info) in effect on the date of publication of this document. Please review these documents carefully, as they describe your rights and restrictions with respect to this document. Code Components extracted from this document must 
include Simplified BSD License text as described in section $4 . e$ of the Trust Legal Provisions and are provided without warranty as described in the Simplified BSD License.

This document may contain material from IETF Documents or IETF Contributions published or made publicly available before November 10, 2008. The person(s) controlling the copyright in some of this material may not have granted the IETF Trust the right to allow modifications of such material outside the IETF Standards Process. Without obtaining an adequate license from the person(s) controlling the copyright in such materials, this document may not be modified outside the IETF Standards Process, and derivative works of it may not be created outside the IETF Standards Process, except to format it for publication as an RFC or to translate it into languages other than English.

Table of Contents

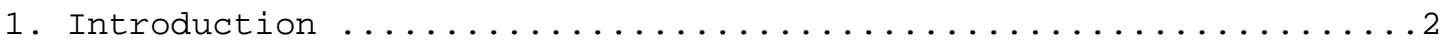

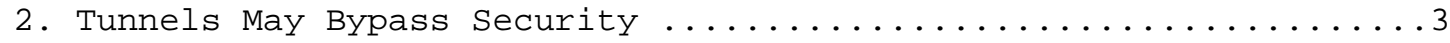

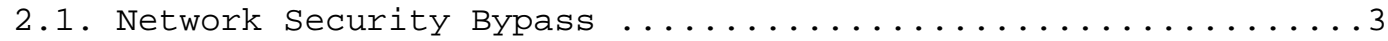

2.2. IP Ingress and Egress Filtering Bypass .............

2.3. Source Routing after the Tunnel client .............6

3. Challenges in Inspecting and Filtering Content of

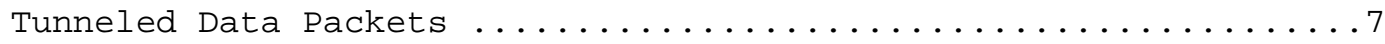

3.1. Inefficiency of Selective Network Filtering of All

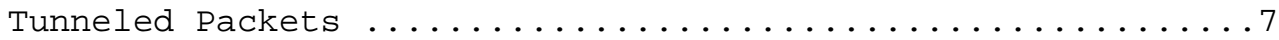

3.2. Problems with Deep Packet Inspection of Tunneled

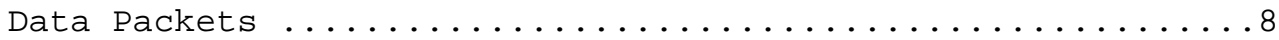

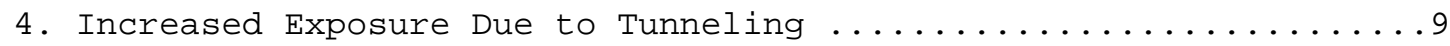

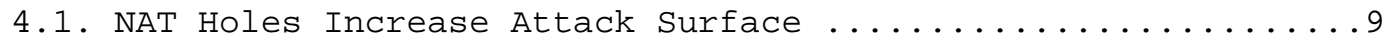

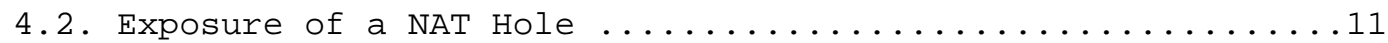

4.3. Public Tunnels Widen Holes in Restricted NATs ..........12

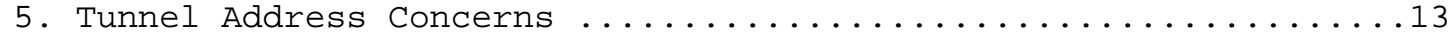

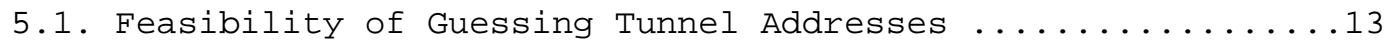

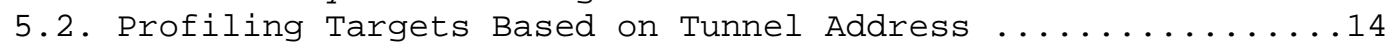

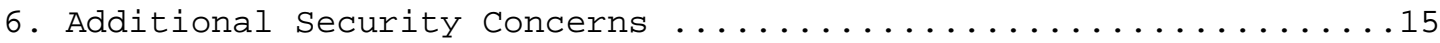
6.1. Attacks Facilitated by Changing Tunnel Server Setting ....15

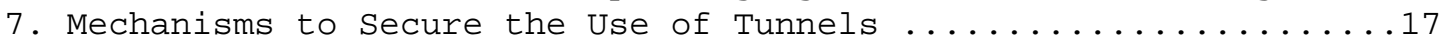

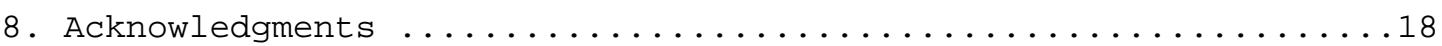

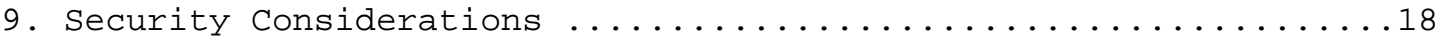

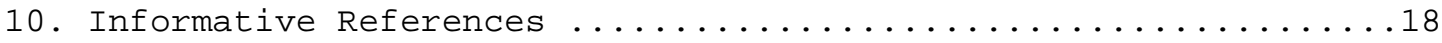




\section{Introduction}

With NAT devices becoming increasingly more prevalent, there have recently been many tunneling protocols developed that go through NAT devices or firewalls by tunneling over UDP or TCP. For example, Teredo [RFC4380], Layer Two Tunneling Protocol Version 2 (L2TPv2) [RFC2661], and Layer Two Tunneling Protocol Version 3 (L2TPv3) [RFC3931] all tunnel IP packets over UDP. Similarly, many secure Socket Layer (SSL) VPN solutions that tunnel IP packets over HTTP (and hence over TCP) are deployed today.

This document discusses security concerns with tunneling IP packets and includes recommendations where relevant.

The primary intent of this document is to help improve security deployments using tunnel protocols. In addition, the document aims to provide information that can be used in any new or updated tunnel protocol specification. The intended audience of this document includes network administrators and future protocol developers.

\section{Tunnels May Bypass Security}

\subsection{Network Security Bypass}

\subsubsection{Problem}

Tunneled IP traffic may not receive the intended level of inspection or policy application by network-based security devices unless such devices are specifically tunnel aware. This reduces defense in depth and may cause security gaps. This applies to all network-located devices and to any end-host-based firewalls whose existing hooking mechanism(s) would not show them the IP packet stream after the tunnel client does decapsulation or before it does encapsulation.

\subsubsection{Discussion}

Evasion by tunneling is often a problem for network-based security devices such as network firewalls, intrusion detection and prevention systems, and router controls. To provide such functionality in the presence of tunnels, the developer of such devices must add support for parsing each new protocol. There is typically a significant lag between when the security developer recognizes that a tunnel will be used (or will be remotely usable) to a significant degree and when the parsing can be implemented in a product update, the update can be tested and released, and customers can begin using the update. Late changes in the protocol specification or in the way it is implemented can cause additional delays. This becomes a significant security concern when a delay in applied coverage is occurring frequently. 
One way to cut down on this lag is for security developers to follow the progress of new IETF protocols, but this will still not account for any new proprietary protocols.

For example, for L2TP or Teredo, an unaware network security device would inspect or regulate the outer IP and the IP-based UDP layer as normal, but it would not recognize that there is an additional IP layer contained inside the UDP payload to which it needs to apply the same controls as it would to a native packet. (Of course, if the device discards the packet due to something in the IP or UDP header, such as referring to an unknown protocol, the embedded packet is no longer a concern.) In addition, if the tunnel does encryption, the network-based security device may not be able to do much, just as if IPsec end-to-end encryption were used without tunneling.

Network security controls not being applied must be a concern to those that set them up, since those controls are supposed to provide an additional layer of defense against external attackers. If network controls are being bypassed due to the use of tunneling, the strength of the defense (i.e., the number of layers of defense) is reduced. Since security administrators may have a significantly reduced level of confidence without this layer, this becomes a concern to them.

One implication of the security control bypass is that defense in depth has been reduced, perhaps down to zero unless a local firewall is in use as recommended in [RFC4380]. However, even if there are host-based security controls that recognize tunnels and all controls that were maintained by the network are available on the host, security administrators may not have configured them with full security control parity. Thus, there may be gaps in desired coverage.

Compounding this is that, unlike what would be the case for native IP, some network administrators will not even be aware that their hosts are globally reachable if the tunnel provides connectivity to/from the Internet; for example, they may not be expecting this for hosts behind a stateful firewall. In addition, Section 3.2 discusses how it may not be efficient to find all tunneled traffic for network devices to examine.

\subsubsection{Recommendations}

Security administrators who do not consider tunneling an acceptable risk should disable tunnel functionality either on the end nodes (hosts) or on the network nodes at the perimeter of their network. However, there may be an awareness gap. Thus, due to the possible negative security consequences, tunneling functionality should be 
easy to disable on the host and through a central management facility if one is provided.

To minimize security exposure due to tunnels, we recommend that a tunnel be an interface of last resort, independent of IP version. Specifically, we suggest that when both native and tunneled access to a remote host is available, the native access be used in preference to tunneled access except when the tunnel endpoint is known to not bypass security (e.g., an IPsec tunnel to a gateway provided by the security administrator of the network). This should also promote greater efficiency and reliability.

Note that although Rule 7 of [RFC3484], Section 6 will prefer native connectivity over tunnels, this rule is only a tie-breaker when a choice is not made by earlier rules; hence, tunneling mechanisms that are tied to a particular range of IP address space will be decided based on the prefix precedence. For example, using the prefix policy mechanism of [RFC3484], Section 2.1, Teredo might have a precedence of 5 so that native IPv4 is preferred over Teredo.

\subsection{IP Ingress and Egress Filtering Bypass}

\subsubsection{Problem}

IP addresses inside tunnels are not subject to ingress and egress filtering in the network they tunnel over, unless extraordinary measures are taken. Only the tunnel endpoints can do such filtering.

\subsubsection{Discussion}

Ingress filtering (sanity-checking incoming destination addresses) and egress filtering (sanity-checking outgoing source addresses) are done to mitigate attacks and to make it easier to identify the source of a packet and are considered to be a good practice. For example, ingress filtering at the network perimeter should not allow packets with a source address that belongs to the network to enter the network from outside the network. This function is most naturally (and in the general case, by requirement) done at network boundaries. Tunneled IP traffic bypassing this network control is a specific case of Section 2.1 , but is illustrative.

\subsubsection{Recommendations}

Tunnel servers can apply ingress and egress controls to tunneled IP addresses passing through them to and from tunnel clients.

Tunnel clients could make an effort to conduct ingress and egress filtering. 
Implementations of protocols that embed an IPv4 address in a tunneled IPV6 address directly between peers should perform filtering based on checking the correspondence.

Implementations of protocols that accept tunneled packets directly from a server, relay, or protocol peer do filtering the same way as it would be done on a native link with traffic from a router.

Some protocols such as 6to4 [RFC3056], Teredo, and the Intra-Site Automatic Tunnel Addressing Protocol (ISATAP) [RFC5214] allow both other hosts and a router over a common tunnel. To perform host-based filtering with such protocols, a host would need to know the outer IP address of each router from which it could receive traffic, so that packets from hosts beyond the router will be accepted even though the source address would not embed the router's IP address. Router addresses might be learned via SEcure Neighbor Discovery (SEND) [RFC3971] or some other mechanism (e.g., [RFC5214], Section 8.3.2).

2.3. Source Routing after the Tunnel Client

\subsubsection{Problem}

If the encapsulated IP packet specifies source routing beyond the recipient tunnel client, the host may forward the IP packet to the specified next hop. This may be unexpected and contrary to administrator wishes and may have bypassed network-based sourcerouting controls.

\subsubsection{Discussion}

A detailed discussion of issues related to source routing can be found in [RFC5095] and [SECA-IP].

\subsubsection{Recommendations}

Tunnel clients should by default discard tunneled IP packets that specify additional routing, as recommended in [RFC5095] and [SECA-IP], though they may also allow the user to configure what source-routing types are allowed. All pre-existing source-routing controls should be upgraded to apply these controls to tunneled IP packets as well. 
3. Challenges in Inspecting and Filtering Content of Tunneled Data Packets

\subsection{Inefficiency of Selective Network Filtering of All Tunneled Packets}

\subsubsection{Problem}

There is no mechanism that both efficiently and immediately filters all tunneled packets (other than the obviously faulty method of filtering all packets). This limits the ability to prevent tunnel use on a network.

\subsubsection{Discussion}

Given concerns about tunnel security or a network's lack of preparedness for tunnels, a network administrator may wish to simply block all use of tunnels that bypass security policies. He or she may wish to do so using network controls; this could be either due to not having the capability to disable tunneling on all hosts attached to the network or due to wanting an extra layer of prevention.

One simple method of doing this easily for many tunnel protocols is to block outbound packets to the UDP or TCP port used (e.g., destination UDP port is 3544 for Teredo, UDP port 1701 for L2TP, etc.). This prevents a tunnel client from establishing a new tunnel. However, existing tunnels will not necessarily be affected if the blocked port is used only for initial setup. In addition, if the blocking is applied on the outside of the client's NAT device, the NAT device will retain the port mapping for the client. In some cases, however, blocking all traffic to a given outbound port (e.g., port 80) may interfere with non-tunneled traffic so this should be used with caution.

Another simple alternative, if the tunnel server addresses are wellknown, is to filter out all traffic to/from such addresses.

The other approach is to find all packets to block in the same way as would be done for inspecting all packets (Section 3.2). However, this presents difficulties in terms of efficiency of filtering, as is discussed in section 3.2 . 


\subsubsection{Recommendations}

Developers of protocols that tunnel over UDP or TCP (including HTTP) to reach the Internet should disable their protocols in networks that wish to enforce security policies on the user traffic. (Windows, for example, disables Teredo by default if it detects that it is within an enterprise network that contains a Windows domain controller.)

Administrators of such networks may wish to filter all tunneled traffic at the boundaries of their networks. It is sufficient to filter out the tunneled connection requests (if they can be identified) to stop further tunneled traffic. The easiest mechanism for this would be to filter out outgoing traffic sent to the destination port defined by the tunneling protocol and incoming traffic with that source port. Similarly, in certain cases, it is also possible to use the IP protocol field to identify and filter tunneled packets. For example, 6to4 [RFC3056] is a tunneling mechanism that uses IPv4 packets to carry encapsulated IPv6 packets and can be identified by the IPv4 protocol type 41.

3.2. Problems with Deep Packet Inspection of Tunneled Data Packets

\subsubsection{Problem}

There is no efficient mechanism for network-based devices, which are not the tunnel endpoint, to inspect the contents of all tunneled data packets the way they can for native packets. This makes it difficult to apply the same controls as they do to native IP.

\subsubsection{Discussion}

Some tunnel protocols are easy to identify, such as if all data packets are encapsulated using a well-known UDP or TCP port that is unique to the protocol.

Other protocols, however, either use dynamic ports for data traffic or else share ports with other protocols (e.g., tunnels over HTTP).

The implication of this is that network-based devices that wish to passively inspect (and perhaps selectively apply policy to) all encapsulated traffic must inspect all TCP or UDP packets (or at least all packets not part of a session that is known not to be a tunnel). This is imperfect since a heuristic must then be applied to determine if a packet is indeed part of a tunnel. This may be too slow to make use of in practice, especially if it means that all TCP or UDP packets must be taken off of the device's "fast path". 
One heuristic that can be used on packets to determine if they are tunnel-related or not is as follows. For each known tunnel protocol, attempt parsing the packet as if it were a packet of that protocol destined to the local host (i.e., where the local host has the destination address in the inner IP header, if any). If all syntax checks pass, up to and including the inner IP header (if the tunnel does not use encryption), then treat the packet as if it were a tunneled packet of that protocol.

It is possible that non-tunneled packets will be treated as if they were tunneled packets using this heuristic, but tunneled packets (of the known types of tunnels) should not escape inspection, absent implementation bugs.

For some protocols, it may be possible to monitor setup exchanges to know to expect that data will be exchanged on certain ports later. (Note that this does not necessarily apply to Teredo, for example, since communicating with another Teredo client behind a cone NAT [RFC5389] device does not require such signaling. In such cases this control will not work. However, deprecation of the cone bit as discussed in [RFC5991] means this technique may indeed work with updated Teredo implementations.)

\subsubsection{Recommendations}

As illustrated above, it should be clear that inspecting the contents of tunneled data packets is highly complex and often impractical. For this reason, if a network wishes to monitor IP traffic, tunneling across, as opposed to tunneling to, the security boundary is not recommended. For example, to provide an IPv6 transition solution, the network should provide native IPv6 connectivity or a tunnel solution (e.g., ISATAP or 6over4 [RFC2529]) that encapsulates data packets between hosts and a router within the network.

\section{Increased Exposure Due to Tunneling}

\subsection{NAT Holes Increase Attack Surface}

\subsubsection{Problem}

If the tunnel allows inbound access from the public Internet, the opening created in a NAT device due to a tunnel client increases its Internet attack surface area. If vulnerabilities are present, this increased exposure can be used by attackers and their programs. 
If the tunnel allows inbound access only from a private network (e.g., a remote network to which one has VPNed), the opening created in the NAT device still increases its attack surface area, although not as much as in the public Internet case.

\subsubsection{Discussion}

When a tunnel is active, a mapped port is maintained on the NAT device through which remote hosts can send packets and perhaps establish connections. The following sequence is intended to sketch out the processing on the tunnel client host that can be reached through this mapped port; the actual processing for a given host may be somewhat different.

1. Link-layer protocol processing

2. (Outer) IP host firewall processing

3. (Outer) IP processing by stack

4. UDP/TCP processing by stack

5. Tunnel client processing

6. (Inner) IP host firewall processing

7. (Inner) IP processing by stack

8. Various upper layer processing may follow

The inner firewall (and other security) processing may or may not be present, but if it is, some of the inner IP processing may be filtered. (For example, [RFC4380], Section 7.1 recommends that an IPv6 host firewall be used on all Teredo clients.)

(By the virtue of the tunnel being active, we can infer that the inner host firewall is unlikely to do any filtering based on the outer IP.) Any of this processing may expose vulnerabilities an attacker can exploit; similarly, these may expose information to an attacker. Thus, even if firewall filtering is in place (as is prudent) and filters all incoming packets, the exposed area is larger than if a native IP Internet connection were in place, due to the processing that takes place before the inner IP is reached (specifically, the UDP/TCP processing, the tunnel client processing, and additional IP processing, especially if one is IPv4 and the other is IPv6). 
One possibility is that a layer 3 (L3) targeted worm makes use of a vulnerability in the exposed processing. The main benefit tunneling provides to worms is enabling L3 reachability to the end host. Even a thoroughly firewalled host could be subject to a worm that spreads with a single UDP packet if the right remote code vulnerability is present.

4.1.3. Recommendation

This problem seems inherent in tunneling being active on a host, so the solution seems to be to minimize tunneling use.

For example, tunneling can be active only when it is really needed and only for as long as needed. So, the tunnel interface can be initially not configured and only used when it is entirely the last resort. The interface should then be deactivated (ideally, automatically) again as soon as possible. Note, however, that the hole will remain in the NAT device for some amount of time after this, so some processing of incoming packets is inevitable unless the client's native IP address behind the NAT device is changed.

\subsection{Exposure of a NAT Hole}

4.2.1. Problem

Attackers are more likely to know about a tunnel client's NAT hole than a typical hole in the NAT device. If they know about the hole, they could try to use it.

\subsubsection{Discussion}

There are at least three reasons why an attacker may be more likely to learn of the tunnel client's exposed port than a typical NAT exposed port:

1. The NAT mapping for a tunnel is typically held open for a significant period of time and kept stable. This increases the chance of it being discovered.

2. In some protocols (e.g., Teredo), the external IP address and port are contained in the client's address that is used end-toend and possibly even advertised in a name resolution system. While the tunnel protocol itself might only distribute this address in IP headers, peers, routers, and other on-path nodes still see the client's IP address. Although this point does not apply directly to protocols that do not construct the inner IP address based on the outer IP address (e.g., L2TP), the inner IP 
address is still known to peers, routers, etc., and can still be reached by attackers without their knowing the external IP address or port.

3. Sending packets over a tunnel often results in more message exchanges due to the tunneling protocol, as well as messages being seen by more parties (e.g., due to a longer path length), than sending packets natively, offering more chances for visibility into the port and address in use.

\subsubsection{Recommendation}

The recommendation from Section 4.1 seems to apply here as well: minimize tunnel use.

4.3. Public Tunnels Widen Holes in Restricted NATs

\subsubsection{Problem}

Tunnels that allow inbound connectivity from the Internet (e.g., Teredo, tunnel brokers, etc.) essentially disable the filtering behavior of the NAT for all tunnel client ports. This eliminates NAT devices filtering for such ports and may eliminate the need for an attacker to spoof an address.

\subsubsection{Discussion}

NATs that implement Address-Dependent or Address and Port-Dependent Filtering [RFC4787] limit the source of incoming packets to just those that are a previous destination. This poses a problem for tunnels that intend to allow inbound connectivity from the Internet.

Various protocols (e.g., Teredo, Session Traversal Utilities for NAT (STUN) [RFC5389], etc.) provide a facility for peers, upon request, to become a previous destination. This works by sending a "bubble" packet via a server, which is passed to the client and then sent by the client (through the NAT) to the originator.

This removes any NAT-based barrier to attackers sending packets in through the client's service port. In particular, an attacker would no longer need to either be an actual previous destination or forge its addresses as a previous destination. When forging, the attacker would have had to learn of a previous destination and then would face more challenges in seeing any returned traffic. 


\subsubsection{Recommendations}

If the tunnel can provide connectivity to the Internet, the tunnel client should run a host firewall on the tunnel interface. Also, minimizing public tunnel use (see Section 4.1.3) would lower the attack opportunity related to this exposure.

5. Tunnel Address Concerns

5.1. Feasibility of Guessing Tunnel Addresses

5.1.1. Problem

For some types of tunneling protocols, it may be feasible to guess IP addresses assigned to tunnels, either when looking for a specific client or when looking for an arbitrary client. This is in contrast to native IPv6 addresses in general but is no worse than for native IPv4 addresses today.

For example, some protocols (e.g., 6to4 and Teredo) use well-defined address ranges. As another example, using well-known public servers for Teredo or tunnel brokers also implies using a well-known address range.

\subsubsection{Discussion}

Several tunnel protocols use endpoint addresses that can be algorithmically derived from some known values. These addresses are structured, and the fields contained in them can be fairly predictable. This reduces the search space for an attacker and reduces the resistance of the address to scanning attacks.

\subsubsection{Recommendations}

It is recommended that tunnel protocol developers use tunnel endpoint addresses that are not easily guessable. When the tunnel endpoint addresses are structured and fairly guessable, it is recommended that the implementation use any unused fields in the address to provide additional entropy to the address in order to reduce the addressscanning risks. For example, this could be done by setting these unused fields to some random values. 


\subsection{Profiling Targets Based on Tunnel Address}

\subsubsection{Problem}

An attacker encountering an address associated with a particular tunneling protocol or well-known tunnel server has the opportunity to infer certain relevant pieces of information that can be used to profile the host before sending any packets. This can reduce the attacker's footprint and increase the attacker's efficiency.

\subsubsection{Discussion}

The tunnel address reveals some information about the nature of the client:

- That a host has a tunnel address associated with a given protocol means that the client is running on some platform for which there exists a tunnel client implementation of that protocol. In addition, if some platforms have that protocol installed by default and if the host's default rules for using it make it susceptible to being in use, then the protocol is more likely to be running on such a platform than on one where it is not used by default. For example, as of this writing, seeing a Teredo address suggests that the host it is on is probably running Windows.

- Similarly, the use of an address associated with a particular tunnel server also suggests some information. Tunnel client software is often deployed, installed, and/or configured using some degree of automation. It seems likely that the majority of the time, the tunnel server that results from the initial configuration will go unchanged from the initial setting. Moreover, the server that is configured for use may be associated with a particular means of installation, which often suggests the platform. For example, if the server field in a Teredo address is one of the IPv4 addresses to which teredo.ipv6.microsoft.com resolves, the host is likely running Windows.

- The external IPv4 address of a NAT device can, of course, be readily associated with a particular organization or at least an ISP; hence, putting this address in an IPV6 address reveals this information. However, this is no different than using a native IP address and is therefore not new with tunneling.

- It is also possible that external client port numbers may be more often associated with particular client software or the platform on which it is running. The usefulness of this for platform determination is, however, reduced by the different NAT port 
number assignment behaviors. In addition, the same observations would apply to use of UDP or TCP over native IP as well; hence, this is not new with tunneling.

The platform, tunnel client software, or organization information can be used by an attacker to target attacks more carefully. For example, an attacker may decide to attack an address only if it is likely to be associated with a particular platform or tunnel client software with a known vulnerability. (This is similar to the ability to guess some platforms based on the Organizationally Unique Identifier (OUI) in the Extended Unique Identifier (EUI)-64 portion of an IPv6 address generated from a Media Access Control (MAC) address, since some platforms are commonly used with interface cards from particular vendors.)

\subsubsection{Recommendations}

If installation programs randomize the server setting, they would reduce the extent to which they can be profiled. Similarly, administrators can choose to change the default setting to reduce the degree to which they can be profiled ahead of time.

Randomizing the tunnel client port in use would mitigate any profiling that can be done based on the external port, especially if multiple tunnel clients did this. Further discussion on randomizing ports can be found at [RFC6056].

It is recommended that tunnel protocols minimize the propagation of knowledge about whether the NAT is a cone NAT.

\section{Additional Security Concerns}

\subsection{Attacks Facilitated by Changing Tunnel Server setting}

\subsubsection{Problem}

If an attacker could change either a tunnel client's server setting or the IP addresses to which a configured host name resolves (e.g., by intercepting DNS queries) AND if the tunnel is not authenticated, the attacker would become a man in the middle. This would allow them to at least monitor peer communication and at worst to impersonate the remote peer.

\subsubsection{Discussion}

A client's server has good visibility into the client's communication with IP peers. If the server were switched to one that records this information and makes it available to third parties (e.g., 
advertisers, competitors, spouses, etc.), then sensitive information would be disclosed, especially if the client's host prefers the tunnel over native IP. Assuming the server provides good service, the user would not have reason to suspect the change.

Full interception of IP traffic could also be arranged (including pharming), which would allow any number of deception or monitoring attacks, including phishing. We illustrate this with an example phishing attack scenario.

It is often assumed that the tunnel server is a trusted entity. It may be possible for malware or a malicious user to quietly change the client's tunnel server setting and have the user be unaware that their trust has been misplaced for an indefinite period of time. However, malware or a malicious user can do much worse than this, so this is not a significant concern. Hence, it is only important that an attacker on the network cannot change the client's server setting.

1. A phisher sets up a malicious tunnel server (or tampers with a legitimate one). This server, for the most part, provides correct service.

2. An attacker, by some means, switches the host's tunnel server setting or spoofs a DNS reply to point to the above server. If neither DNS nor the tunnel setup is secured (i.e., if the client does not authenticate the information), then the attacker's tunnel server is seen as legitimate.

3. A user on the victim host types their bank's URL into his/her browser.

4. The bank's hostname resolves to one or more IP addresses, and the tunnel is selected for socket connection for whatever reason (e.g., the tunnel provides IPv6 connectivity, and the bank has an IPv6 address).

5. The tunnel client uses the server for help in connecting to the bank's IP address. Some tunneling protocols use a separate channel for signaling versus data, but this still allows the server to place itself in the data path by an appropriate signal to the client. For example, in Teredo, the client sends a ping request through a server, which is expected to come back through a data relay, and a malicious server can simply send it back itself to indicate that is a data relay for the communication. 
6. The rest works pretty much like any normal phishing transaction, except that the attacker acts as a tunnel server (or data relay, for protocols such as Teredo) and a host with the bank's IP address.

This pharming-type attack is not unique to tunneling. Switching DNS server settings to a malicious DNS server or DNS cache poisoning in a recursive DNS resolver could have a similar effect.

\subsubsection{Recommendations}

In general, anti-phishing and anti-fraud provisions should help with aspects of this, as well as software that specifically monitors for tunnel server changes.

Perhaps the best way to mitigate tunnel-specific attacks is to have the client authenticate either the tunnel server or at least the means by which the tunnel server's IP address is determined. For example, SSL VPNs use https URLs and hence authenticate the server as being the expected one. When IPv6 Router Advertisements are sent over the tunnel, another mechanism is to use sEcure Neighbor Discovery (SEND) [RFC3971] to verify that the client trusts the server.

On the host, it should require an appropriate level of privilege in order to change the tunnel server setting (as well as other nontunnel-specific settings such as the DNS server setting, etc.). Making it easy to see the current tunnel server setting (e.g., not requiring privilege for this) should help detection of changes.

The scope of the attack can also be reduced by limiting tunneling use in general but especially in preferring native IPv4 to tunneled IPv6 when connecting to peers who are accessible over IPv4, as doing so helps mitigate attacks that are facilitated by changing the tunnel server setting. Please refer to section 3 of [TUNNEL-LOOPS] for a detailed description and mitigation measures for a class of attacks based on IPv6 automatic tunnels.

7. Mechanisms to Secure the Use of Tunnels

This document described several security issues with tunnels. This does not mean that tunnels need to be avoided at any cost. On the contrary, tunnels can be very useful if deployed, operated, and used properly. The threats against IP tunnels are documented here. If the threats can be mitigated, network administrators can efficiently and securely use tunnels in their network. Several measures can be taken in order to secure the operation of IPv6 tunnels: 
- Operating on-premise tunnel servers/relays so that the tunneled traffic does not cross border routers.

o Setting up internal routing to steer traffic to these servers/ relays

o Setting up of firewalls [RFC2979] to allow known and controllable tunneling mechanisms and disallow unknown tunnels.

8. Acknowledgments

The authors would like to thank Remi Denis-Courmont, Fred Templin, Jordi Palet Martinez, James Woodyatt, Christian Huitema, Brian Carpenter, Nathan Ward, Kurt Zeilenga, Joel Halpern, Erik Kline, Alfred Hoenes, and Fernando Gont for reviewing earlier versions of the document and providing comments to make this document better.

9. Security Considerations

This entire document discusses security concerns with tunnels.

10. Informative References

[RFC2529] Carpenter, B. and C. Jung, "Transmission of IPv6 over IPv4 Domains without Explicit Tunnels", RFC 2529, March 1999.

[RFC2661] Townsley, W., Valencia, A., Rubens, A., Pall, G., Zorn, G., and B. Palter, "Layer Two Tunneling Protocol "L2TP"", RFC 2661, August 1999.

[RFC2979] Freed, N., "Behavior of and Requirements for Internet Firewalls", RFC 2979, October 2000.

[RFC3056] Carpenter, B. and K. Moore, "Connection of IPv6 Domains via IPv4 Clouds", RFC 3056, February 2001.

[RFC3484] Draves, R., "Default Address Selection for Internet Protocol version 6 (IPv6)", RFC 3484, February 2003.

[RFC3931] Lau, J., Ed., Townsley, M., Ed., and I. Goyret, Ed., "Layer Two Tunneling Protocol - Version 3 (L2TPv3)", RFC 3931, March 2005.

[RFC3971] Arkko, J., Ed., Kempf, J., Zill, B., and P. Nikander, "SEcure Neighbor Discovery (SEND)", RFC 3971, March 2005. 
[RFC4380] Huitema, C., "Teredo: Tunneling IPv6 over UDP through Network Address Translations (NATs)", RFC 4380, February 2006 .

[RFC4787] Audet, F., Ed., and C. Jennings, "Network Address Translation (NAT) Behavioral Requirements for Unicast UDP", BCP 127, RFC 4787, January 2007.

[RFC5095] Abley, J., Savola, P., and G. Neville-Neil, "Deprecation of Type 0 Routing Headers in IPv6", RFC 5095, December 2007 .

[RFC5214] Templin, F., Gleeson, T., and D. Thaler, "Intra-Site Automatic Tunnel Addressing Protocol (ISATAP)", RFC 5214, March 2008 .

[RFC5389] Rosenberg, J., Mahy, R., Matthews, P., and D. Wing, "Session Traversal Utilities for NAT (STUN)", RFC 5389, October 2008 .

[RFC5991] Thaler, D., Krishnan, S., and J. Hoagland, "Teredo Security Updates", RFC 5991, September 2010.

[RFC6056] Larsen, M. and F. Gont, "Recommendations for TransportProtocol Port Randomization", BCP 156, RFC 6056, January 2011.

[SECA-IP] Gont, F., "Security Assessment of the Internet Protocol version 4", Work in Progress, April 2011.

[ TUNNEL-LOOPS ]

Nakibly, G. and F. Templin, "Routing Loop Attack using IPv6 Automatic Tunnels: Problem Statement and Proposed Mitigations", Work in Progress, March 2011. 


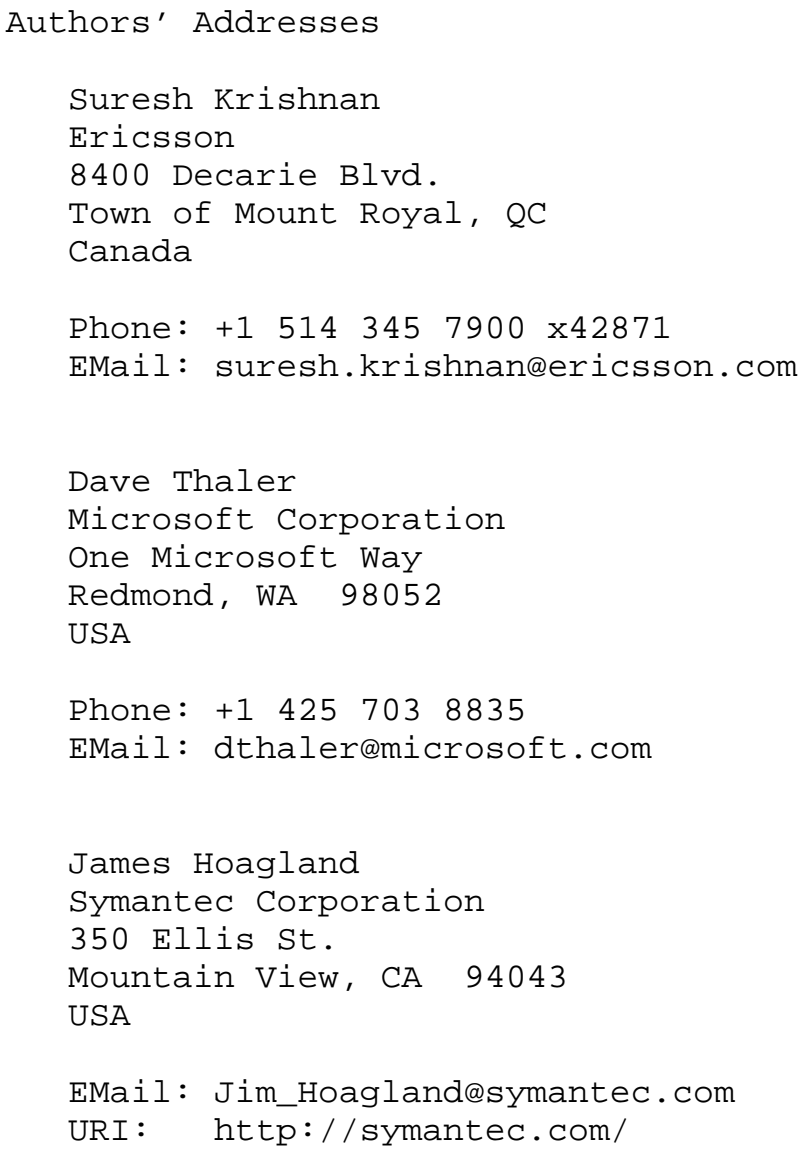

